\title{
Relationships of Colorectal Cancer with Dietary Factors and Public Health Indicators: an Ecological Study
}

\author{
Hedayat Abbastabar ${ }^{1}$,Abazar Roustazadeh ${ }^{2}$,Ali Alizadeh ${ }^{3 *}$, Parvin Hamidifard ${ }^{4}$, \\ Mehrdad Valipour ${ }^{5}$, Ali Asghar Valipour ${ }^{6}$
}

\begin{abstract}
Background: Colorectal cancer (CRC) is the third most common cancer in Iranian women and fifth in men. The aims of this study were to investigate the relation of dietary factors and public health indicators to its development. Materials and Methods: The required information (2001-2006) about risk factors was obtained from the NonCommunicable Disease Surveillance Centre (NCDSC) of Iran. Risk factor data (RFD) from 89,404 individuals (15-64 years old) were gathered by questionnaire and laboratory examinations through a cross sectional study in all provinces by systematic clustering sampling method. CRC incidence segregated by age and gender was obtained from Cancer Registry Ministry of Health (CRMH) of Iran. First, correlation coefficients were used for data analysis and then multiple regression analysis was performed to control for confounding factors. Results: Colorectal cancer incidence showed a positive relationship with diabetes mellitus, hypertension, lacking or low physical activity, high education, high intake of dairy products, and non-consumption of vegetables and fruits. Conclusions: We concluded that many dietary factors and public health indicators have positive relationships with CRC and might therefore be targets of preliminary prevention. However, since this is an ecological study limited by potential ecological fallacy the results must be interpreted with caution.
\end{abstract}

Keywords: Colorectal cancer, ecologic study - risk factor - developing countries - correlation

Asian Pac J Cancer Prev, 16 (9), 3991-3995

\section{Introduction}

Nowadays, colorectal cancer (CRC) is one of the most important problems of public health throughout the world. Every year almost one million new cases of colorectal cancer are diagnosed and near half of the cases will die (Ansari et al., 2006; Erichsen et al., 2013; Jess et al., 2013). CRC is the fourth common cancer in men and third in women (Boyle et al., 2002). The cumulative incidence of $\mathrm{CRC}$ in western European and northern American countries, and Middle East countries is 30-50 and 3-7 cases per 100000 persons, respectively (Hind et al., 2008; Elsamany et al., 2014).

In European and Middle East countries only 2-8 and $15-35 \%$ of CRC cases occurred in persons lower than forty years old, respectively (Hind et al., 2008; Gruenberger et al., 2008). Iranian cancer registry reported that CRC is the third common cancer in women and fifth in men (Iranian Annual Cancer Report, 2005-2007) which is the same as the results of globocan report(Globocan, 2008).

Also globocan report showed that colorectal cancer is the 4th common cancer in Iran. In addition, CRC is the 3rd place for many Mediterranean countries(Akhavan et al., 2014). It is the 5th and 3rd common cancer in men and women, respectively (Globocan, 2008). Prevalence of CRC is almost the same in Iran and Mediterranean countries.

At first, CRC was widespread in advanced countries because the risk factors were prevalent there, but recently CRC communed in developing countries that had low risk factors (Caygill et al., 1996; Chao et al., 2005; Baghestani et al., 2014).

CRC has a positive relationship with obesity, diet with high rate of fats and low vegetables, non physical activity and smoking (Fernandez et al., 2002; Hjartåker et al., 2013; Robsahm et al., 2013). The role of nutrition factors was explored in many studies but the results are inconsistent with each other (Caygill et al., 1996; Coups et al., 2007). Incidence of CRC is high in countries with high rates of meat consumption (Kim et al., 2013; Fateh, 2008).

Since the intake of vegetables, fruits and olive oil are higher in Mediterranean area so the incidence of CRC in these area countries is lower than western countries (Bamia et al., 2013). Furthermore, studies showed that the

\footnotetext{
${ }^{1}$ Department of Epidemiology, Schools of Health and Nutrition, Shiraz University of Medical Sciences, Shiraz, ${ }^{2}$ Department of Biochemistry and Research Center for Non-communicable Disease, Jahrom University of Medical Sciences, Jahrom, ${ }^{3}$ Research Center for Social Determinants in Health Promotion, Department of Research and Technology, Hormozgan University of Medical Sciences, Bandar-e-Abbas, ${ }^{4}$ Tehran University of Medical Sciences, Tehran, ${ }^{5}$ Lorestan University of Medical Sciences, Khorram Abad, ${ }^{6}$ Ahvaz Jundishapur University of Medical Sciences, Ahvaz, Iran *For correspondence: Ali.Alizadeh@hums.ac.ir
} 
secondary density of bilious acid is related to increase of CRC incidence (Kamano et al., 1999).

Also, nutrition factors including meat, fat, cereal, vegetables, fruits, milk and olive oil can influence the duration time transfer of colon and secondary density of bilious acid (Dominianni et al., 2013). Nowadays, evidence showed that $\mathrm{CRC}$ is a genetic disease and 10$15 \%$ of CRC cases have genetic cause so that one out of 200 individuals has genetic high risk allele for CRC (Fernandez et al., 2002; Turati et al., 2013; Han et al., 2014).

One third of all cancer cases could be attributed in dietary factors and physical activity, whereas $25 \%$ of CRC could have been avoided only by the adherence to Mediterranean diet (Trichopoulou et al., 2000). Totally, $\mathrm{CRC}$ is more common in older person e.g. the incidence of CRC in western countries among the persons who were less and upper than 65 years old was 20 and 237 per 100000 , respectively. The incidence of CRC is almost the same in men and women (Stoneham et al., 2000; Kushi et al., 2002).

Because of CRC importance and its high incidence, the aims of this study were to determine the relation of nutrition factors including consumption of vegetables, fruits, dairy products, fried foods and salt, and to explore the role of public health indicators including BMI and BMI category classification, physical activity, hypertension, diabetes mellitus, smoking habits, education and marital status on the developing of CRC in Iran.

\section{Materials and Methods}

\section{Data collection}

This is an ecologic study that the required information (2001-2006) about risk factors was obtained from NonCommunicable Disease Surveillance Centre (NCDSC) of Iran. Important risk factors assessed in this study were including dietary factors such as consumption of vegetable, fruits, dairy products, fried foods and salt .Also public health indicators including BMI, physical activity, hypertension, diabetes mellitus, cigarette smoking, education and marital status were investigated.

Risk factor data (RFD) from 89404 individuals (15-64 years old) were gathered by a questionnaire and laboratory examinations through a cross sectional study in all provinces of Iran. RFD was acquired by systematic clustering sampling method and the proportion of clusters were related to the number of household in different rural and urban areas.

Information about the variables associated with dietary factors such as consumption of vegetables, fruits, salt, etc were collected by questionnaire as self reported but the information of biochemical parameters such as blood sugar, blood fat and etc were obtained through blood tests. In addition, Questionnaire was contained questions about demographic variables such as sex, education, marital status and consumption of various foods per week e.g. dairy products, fruits, and etc. We used National NCD Risk Factor Survey Instrument questionnaire from WHO that had sufficient validity and reliability.

For gathering data from different provinces, the experts were trained how to fill out the questionnaire and then they organized workshops for their co-workers. Persons were used to complete the questionnaire working as diseases expert fighters in the health care system of Iran.

Risk factors were classified as Hypertension (SBP/ DBP $>140 / 90 \mathrm{mmHg}$ ), Diabetes Mellitus (FBS $\geq 126$ $\mathrm{mg} / \mathrm{dl}$ ), Education( illiteracy, elementary or high school, and academic education), Cigarette smoking status( none smoker, ex- smoker and current smoker), Marital status( married, single and divorced). Also, intake of vegetable, fruits, fried foods and dairy products were categorized into two strata; persons never consume and who consume these foods weekly.

In addition, intake of salt per day was categorized into three strata; persons never, one and two or more times. Physical activity was including persons with low physical activity (individuals who not meet criteria for moderate or high Categories), persons with moderate physical activity (three or more days of vigorous-intensity activity of at least twenty minutes/week) and persons with severe physical activity (six or more days of any combination of walking, moderate-intensity/week). Finally, Weight (BMI) was computed as a continuous variable in each gender.

However, CRC incidence segregated by age and gender was obtained from Cancer Registry Ministry of Health (CRMH) of Iran.

\section{Statistical analysis}

At first, the correlation coefficient between incidence of CRC and prevalence of each factor was calculated. We calculate Kendal correlation for ordinate scale variables (e.g. smoking) and Pierson correlation for continuous variables (e.g. BMI). Afterward, the factors had statistically significant correlation coefficient ( $p$-value $<0.2$ ) were entered in multiple linear regression model for controlling confounding effect. For statistical analysis we used SPSS software, version 18 , and p-value $<0.2$ for correlation coefficient and $<0.05$ for linear regression coefficients considered as significance levels.

\section{Results}

A significant negative relationship was seen between $\mathrm{CRC}$ and non-consumption of dairy products and intake of fruits weekly in men. Also, CRC showed a significant relationship with non-consumption of vegetables and fruits, intake of dairy products and body mass index (BMI) (Table 1).

Results indicated a significant negative relationship between CRC and illiteracy, non-consumption of dairy products weekly, intake of vegetables and fruits in women. However, CRC showed a significant positive relationship with hypertension, diabetes mellitus, academic education, non-consumption of vegetables and fruits, non- and low physical activity, intake of dairy products and women body mass index (BMI) (Table 1).

In men and women (among factors that had positive relationship) BMI and non physical activity had the highest correlation coefficient, respectively. In contrast, among factors had negative relationship, intake of vegetables and fruits showed the biggest correlation coefficients in both 
Table 1. Correlation Coefficient of Colorectal Cancer Incidence in Iranian Men and Women

\begin{tabular}{lrc}
\hline Variables & $\begin{array}{c}\text { Women } \\
\text { correlation } \\
\text { coefficents }\end{array}$ & $\begin{array}{c}\text { Men } \\
\text { correlation } \\
\text { coefficents }\end{array}$ \\
\hline Hypertention & $* 0.467$ & $\mathrm{NS}$ \\
Diabetes & $* 0.462$ & $\mathrm{NS}$ \\
Body mass index & $* 0.413$ & $* * 0.685$ \\
Non-intake of vegetables weekly & $* 0.367$ & $\mathrm{NS}$ \\
Non-intake of fruits weekly & $* 0.450$ & $* * 0.541$ \\
Non-physical activity weekly & $* 0.470$ & $\mathrm{NS}$ \\
Low physical activity weekly & $* 0.390$ & $\mathrm{NS}$ \\
Intake of dairy products weekly & $* 0.428$ & $* 0.434$ \\
Illiteracy & $*-0.462$ & $\mathrm{NS}$ \\
Non-intake of dairy products weekly & $*-0.404$ & $* *-0.485$ \\
Intake of vegetables weekly & $* 0.432$ & $* *-0.491$ \\
Intake of fruits weekly & $*-0.418$ & $* *-0.534$ \\
\hline
\end{tabular}

**correlation is significant at the 0.01 level (2-tailed); *correlation is significant at the 0.05 level (2-tailed); NS: not significant

Table 2. Multiple Linear Regressions Analysis of Colorectal Cancer Incidence in Iranian Men

\begin{tabular}{lrrrrr}
\hline Variables & \multicolumn{2}{c}{$\begin{array}{c}\text { Unadjusted } \\
\text { coefficients }\end{array}$} & & $\begin{array}{c}\text { Adjusted } \\
\text { coefficients }\end{array}$ & $\mathrm{p}$ value \\
\cline { 2 - 3 } & \multicolumn{1}{c}{$\mathrm{B}$} & $\mathrm{SE}$ & $\mathrm{B}$ & \\
\hline Intercept & -19.095 & 7.249 & & 0.015 \\
Men BMI & 1.115 & 0.278 & 0.563 & 0.001 \\
intake of fruits weekly & -0.163 & 0.071 & -0.333 & 0.031 \\
\hline
\end{tabular}

Table 3. Multiple Linear Regressions Analysis of Colorectal Cancer Incidence in Iranian Women

\begin{tabular}{lrrrrr}
\hline Variables & \multicolumn{2}{c}{$\begin{array}{l}\text { Unadjusted } \\
\text { coefficients }\end{array}$} & \multicolumn{2}{c}{$\begin{array}{c}\text { Adjusted } \\
\text { coefficients }\end{array}$} & $\mathrm{p}$ value \\
\cline { 2 - 3 } & $\mathrm{B}$ & $\mathrm{SE}$ & $\mathrm{B}$ & \\
\hline Intercept & 1.907 & 1.372 & & 0.018 \\
Illiteracy & -0.040 & 0.014 & -0.365 & 0.008 \\
Intake of fruits weekly & -0.145 & 0.048 & -0.357 & 0.006 \\
Hypertension & 0.435 & 0.154 & 0.330 & 0.011 \\
Women BMI & 0.013 & 0.005 & 0.327 & 0.012 \\
Diabetes mellitus & 0.224 & 0.012 & 0.299 & 0.024 \\
\hline
\end{tabular}

the men and women (Table 1).

Results of multiple linear regressions in men showed that CRC was only related to BMI, intake of vegetables and fruits weekly (Table 2), but in women, CRC was related to illiteracy, intake of vegetables and fruits weekly, hypertension, diabetes mellitus and BMI (Table 3). Also smoking showed no association with CRC incidence in men and women.

\section{Discussion}

We explored relationship of CRC incidence with most common non-communicable disease risk factors. Regression analysis findings showed that CRC is related to BMI and intake of vegetables and fruits in men. Also CRC is related to illiteracy, intake of vegetables and fruits, hypertension, diabetes mellitus and BMI in women (Tables 2,3).

Heshmati et.al showed that the mean of BMI was 26.13 and 28.86 , and the prevalence of obesity was $18.6 \%$
Colorectal Cancer, Dietary Factors and Public Health Indicators and $38.3 \%$ in men and women, respectively. (Heshmati et al., 2013). Another study conducted in Iran reported that the prevalence of diabetes mellitus, hypertension, obesity, consumption of fruit, vegetables and fish in East Azerbaijan province was 5.5\%, 16.3\%, 17\%, 95.6\%, $83 \%$ and $29.7 \%$, respectively (Taghavidoost et al., 2011). However, World Health Organization showed that the prevalence of obesity, overweight, diabetes mellitus and high cholesterol in Philippines is 4.8\%, 19.6\%, 4.6\% and $8.5 \%$, respectively (WHO). Comparisons the abovementioned factors in Iran and Philippines suggest that the prevalence rate of most causes are higher in Iran than Philippines (WHO).

Some studies found a positive relationship between CRC incidence and socioeconomic development (Quarini et al., 2002; Levin et al., 2008). Since Iran has recently elapsed to such developments so the positive relationship between CRC and academic education in women could be attributed to positive confounding of socioeconomic status (SES) because Iranian women with higher education usually had better SES. These findings are consistent with other studies (Levin et al., 2008).

The negative relationship of CRC incidence with intake of vegetables, fruits and physical activity was contradicted with other studies (Caygill et al., 1996; Stoneham et al., 2000; Riboli et al., 2003; Granci et al., 2013). It is logical to assume that the fiber of fruits and vegetables decreasing the density of fecal carcinogens and reducing the contact time of carcinogens with the gastrointestinal tract could reduce the incidence of CRC.

Furthermore, some fruits (e.g. orange and lemon) and vegetables have $\mathrm{B}$ and $\mathrm{C}$ vitamins, and antioxidant elements which decrease the CRC incidence (Stoneham et al., 2000; Gluecker et al., 2002; Smith et al., 2007; Center et al., 2009).

However, results showed a positive relationship between CRC incidence and intake of dairy products. Some studies showed a positive relationship between fatty foods and CRC incidence. (Stoneham et al., 2000; Satia-Abouta et al., 2003; Pais et al., 2009). Dairy products e.g. yogurt and butter are rich in saturated fat, so this relationship might be due to the content of fat in these products.

In addition, CRC incidence and obesity showed a positive relationship with diabetes mellitus and hypertension in women. Other studies reported that overweight and obesity are risk factors for CRC incidence (Steindorf et al., 2005; Pais et al., 2009; Wu et al., 2012; Smiechowski et al., 2013). Our finding should interpret with caution because these relations might happen by positive confounding of obesity.

We hope to reduce the incidence of CRC morbidity and mortality through the appropriate interventions like education programs in public schools and broadcasting sensitive people about the risk factors. Issues that need to be included in the training program summarized as: $i$ ) diet containing fruits and vegetables, $i i$ ) reducing consumption of fatty foods, iii) physical activity to prevent obesity, diabetes mellitus and hypertension.

Limitation: Ecological studies are valuable for the estimation of the incidence of a disease, in this case of 
$\mathrm{CRC}$, can show several associations but are not able to indicate whether the investigated factors (e.g. dietary factors) are harmful or protective to the investigated disease. This type of study can only set several hypotheses that need to be further investigated.

In conclusion, many dietary factors and public health indicators that showed a positive relation to CRC are responses to preliminary prevention, so other studies are needed to define subtle roles of them on CRC. Because these factors are constellation variable and the relationships might be occurred by other unmeasured variables so the results must be explained with caution..

\section{References}

Akhavan A, Binesh F, Soltani A (2014). Survival of rectal cancer in Yazd, Iran. Asian Pac J Cancer Prev, 15, 4857-60.

Ansari R, Mahdavinia M, Sadjadi A, et al (2006). Incidence and age distribution of colorectal cancer in Iran: Results of a population-based cancer registry. Cancer Lett, 240, 143-7.

Baghestani AR, Daneshvar T, Pourhoseingholi MA, et al (2014). Survival of colorectal cancer patients in the presence of competing-risk. Asian Pac J Cancer Prev, 15, 6253-5.

Bamia C, Lagiou P, Buckland G, et al (2013). Mediterranean diet and colorectal cancer risk: results from a European cohort. Eur J Epidemiol, 28, 317-28.

Boyle P, Leon ME (2002). Epidemiology of colorectal cancer. Br Med Bull, 64, 1-25.

Caygill CP, Charlett A, Hill MJ (1996). Fat, fish, fish oil and cancer. Br J Cancer, 74, 159-64.

Center MM, Jemal A, Smith RA, et al (2009). Worldwide variations in colorectal cancer. CA Cancer J Clin, 59,366-78.

Chao A, Thun MJ, Connell CJ, et al (2005). Meat consumption and risk of colorectal cancer. JAMA, 293, 172-82.

Coups EJ, Manne SL, Meropol NJ, et al (2007). Multiple behavioral risk factors for colorectal cancer and colorectal cancer screening status. Cancer Epidemiol Biomarkers Prev, 16, 510-6.

Dominianni C, Huang WY, Berndt S, et al (2013). Prospective study of the relationship between coffee and tea with colorectal cancer risk: The PLCO CancerScreening Trial. Br J Cancer, 109, 1352-9.

Elsamany SA, Alzahrani AS, Mohamed MM, et al (2014). Clinico-pathological patterns and survival outcome of colorectal cancer in young patients: western Saudi Arabia experience. Asian Pac J Cancer Prev, 15, 5239-43.

Erichsen R, Horváth-Puhó E, H Iversen L, et al (2013). Does comorbidity interact with colorectal cancer to increase mortality? A nationwide population-based cohort study. $\mathrm{Br}$ J Cancer, 109, 2005-13.

Fateh S (2008). An epidemiologic study of colorectal cancer in Arak during 1994-2004. Iranian Surgeon Megazin, 16 (in Persian).

Fernandez E, La Vecchia C, Talamini R, et al (2002). Joint effects of family history and adult life dietary risk factors on colorectal cancer risk. Epidemiology, 13, 360-3.

Globocan (2008). Available at: http://globocan.iarc.fr/factsheet. aspx.

Gluecker T, Dorta G, Keller W, et al (2002). Performance of multidetector computed tomography colonography compared with conventional colonoscopy. Gut, 51, 207-11.

Granci V, Cai F, Lecumberri E, et al (2013). Colon cancer cell chemosensitisation by fish oil emulsion involves apoptotic mitochondria pathway. Br J Nutr, 109, 1188-95.

Gruenberger B, Tamandl D, Schueller J, et al (2008). Bevacizumab, capecitabine, and oxaliplatin as neoadjuvant therapy for patients with potentially Curable metastatic colorectal cancer. J Clin Oncol, 26, 1830-5.

Han Y, Xue XF, Shen HG, et al (2014). Prognostic significance of Beclin-1 expression in colorectal cancer: a meta-analysis. Asian Pac J Cancer Prev, 15, 4583-7.

Heshmati R, Fakhrzadeh H, Porebrahim R, et al (2013). Assessment of obesity and overweight patterns and changes in the population aged 25-64 population research center tehran university of medical sciences. J Diabetes and Lipid Disorders, 3, 63-70. (in Persian)

Higuchi T, Jass JR (2004). My approach to serrated polyps of the colorectum. J Clin Pathol, 57, 682-6.

Hind D, Tappenden P, Tumur I, et al (2008). The use of irinotecan, oxaliplatin and raltitrexed for the treatment of advanced colorectal cancer: systematic review and economic evaluation. Health Technol Assess, 12, 162.

Hjartåker A, Aagnes B, Robsahm TE, et al (2013). Subsitespecific dietary risk factors for colorectal cancer: a review of cohort studies. J Oncol, 2013, 703854.

Iran Ministry of Health (2008). Annual cancer report. 2005-2007 (in Persian).

Jess T, Horváth-Puhó E, Fallingborg J, et al (2013). Cancer risk in inflammatory bowel disease according to patient phenotype and treatment: a danish population-based cohort study. Am J Gastroenterol, 108, 1869-76.

Kamano T, Mikami Y, Kurasawa T, et al (1999). Ratio of primary and secondary bile acids in feces: possible marker for colorectal cancer? Dis Colon Rectum, 42, 668-72.

Kim E, Coelho D, Blachier F (2013). Review of the association between meat consumption and risk of colorectal cancer. Nutr Res, 33, 983-94.

Kushi L, Giovannucci E (2002). Dietary fat and cancer. Am J Med, 113, 63-70.

Levin B, Lieberman DA, McFarland B, et al (2008). Screening and surveillance for the early detection of colorectal cancer and adenomatous polyps, 2008: a joint guideline from the American Cancer Society, the US multi-society task force on colorectal cancer, and the American college of radiology. Gastroenterology, 134, 1570-95.

Pais R, Silaghi H, Silaghi AC, et al (2009). Metabolic syndrome and risk of subsequent colorectal cancer. World $J$ Gastroenterol, 15, 5141-8.

Quarini C, Gosney M (2002). Review of the evidence for a colorectal cancer screening programme in elderly people. Age Ageing, 38, 503-8.

Riboli E, Norat T (2003). Epidemiologic evidence of the protective effect of fruit and vegetables on cancer risk. Am J Clin Nutr, 78, 559-69.

Robsahm TE, Aagnes B, Hjartåker A, et al (2013). Body mass index, physical activity, and colorectal cancer by anatomical subsites: a systematic review and meta-analysis of cohort studies. Eur J Cancer Prev, 22, 492-505.

Satia-Abouta J, Galanko JA, Potter JD, et al (2003). Associations of total energy and macronutrients with colon cancer risk in African Americans and Whites: results from the North Carolina colon cancer study. Am J Epidemiol, 158, 951-62.

Smiechowski B, Azoulay L, Yin H, et al (2013). The use of metformin and colorectal cancer incidence in patients with type ii diabetes mellitus. Cancer Epidemiol Biomarkers Prev, 22, 1877-83.

Smith RA, Cokkinides V, Eyre HJ (2007). Cancer screening in the United States, 2007: a review of current guidelines, practices, and prospects. CA Cancer J Clin, 57, 90-104.

Steindorf K, Jedrychowski W, Schmidt M, et al (2005). Case control study of lifetime occupational and recreational physical activity and risks for colon and rectal cancer. Eur J Cancer Prev, 14, 363-71. 
Stoneham M, Goldacre M, Seagroatt V, et al (2000).Olive oil, diet and colorectal cancer: an ecological study and a hypothesis. J Epidemiol Community Health, 54, 756-60.

Taghavidoost N, Dastgiri V (2011). Comparison of risk factors for non-communicable diseases in East Azerbaijan Province. Urmia Medical Journal, 22, 512-20 (article in Persian).

Trichopoulou A, Lagiou P, Kuper H, et al (2000). Cancer and mediterranean dietary traditions. Cancer Epidemiol Biomarkers Prev, 9, 869-73.

Turati F, Edefonti V, Bosetti C, et al (2013). Family history of cancer and the risk of cancer: a network of case-control studies. Ann Oncol, 24, 2651-6.

World Health Organization (WHO). Overview of Major Noncommunicable Disease (NCD). Available at: http://www. wpro.who.int/philippines/publications/module1.pdf.

Wu S, Feng B, Li K, et al (2012). Fish consumption and colorectal cancer risk in humans: a systematic review and meta-analysis. Am J Med, 125, 551-9. 